\title{
Tamaño del Estado (gasto público) y salud en el mundo, 1990-2000
}

\author{
Álvaro Franco ${ }^{\mathrm{a}}$ / Diana Gil ${ }^{\mathrm{b}}$ / Carlos Álvarez-Dardet ${ }^{\mathrm{c}}$ \\ aObsevatorio de Políticas Públicas y Salud (OPPS). Universidad de Alicante. Alicante. España. \\ Universidad de Antioquia. Facultad Nacional de Salud Pública. Colombia. \\ bUniversidad de Alicante. Alicante. España. \\ universidad de Alicante. Alicante. España.
}

(Size of the State (public expenditure) and health in a sample of countries worldwide, 1990-2000)

Resumen
Objetivo: Determinar la relación entre el tamaño del Esta-

do (medido como el gasto público) y los indicadores de salud en una muestra de países de todas las regiones del mundo, en la década 1990-2000.

Métodos: Se realizó un estudio ecológico a partir de datos sobre gasto gubernamental central (GGC) y producto interior bruto (PIB) per cápita, obtenidos del Fondo Monetario Internacional, y de esperanza de vida, mortalidad materna y mortalidad infantil, de la Organización Mundial de la Salud. Se construyó un modelo de regresión lineal múltiple para estimar el efecto del GGC sobre salud, introduciendo también el PIB per cápita y la región.

Resultados: EI GGC varía poco durante el período estudiado y converge alrededor de una media del $28 \%$, pero aún dentro de un amplio rango (7,80-53,0\%); los países con economías altas (según PIB per cápita) presentan mayor GGC. Su efecto tiene especial importancia en la mortalidad infantil $(r$ $=0,40$; beta $=-1,327 ; \mathrm{EE}=0,237 ; \mathrm{t}=-5,590 ; \mathrm{p}<0,001$ ). También el PIB per cápita y la ubicación regional se asocian con los cambios en la salud y coinciden con el empeoramiento de los indicadores de salud en algunos países pobres de África y Asia. En el modelo ajustado, el GGC mantiene la asociación estadística con la mortalidad infantil y materna.

Conclusión: El estudio sugiere un efecto importante del tamaño del Estado en la situación de la salud, específicamente en la mortalidad. Aunque tienen limitaciones, dada la reducida ventana temporal utilizada, estos resultados deben ser considerados en el debate político y epidemiológico actual. Palabras clave: Salud. Estado. Gasto público. Globalización. Estudio ecológico.

\begin{abstract}
Objective: To determine the relationship between central government expenditure (CGE), as an expression of the size of the state, and a selection of health indicators in a sample of states worldwide from 1990 to 2000.

Methods: An ecological study was performed using data on CGE and per capita gross national product (GNP), obtained from the official statistics of the International Monetary Fund, and data on life expectancy, maternal and infant mortality, obtained from the World Health Organization. We fitted a multiple regression model to adjust for the effect of CGE on health. Per capita GNP and region were also introduced into the model.
\end{abstract}

Results: The CGE varied little during the study period, converging around a mean of $28 \%$, but within a wide range (min.7.80\%, max. 53.50\%). The countries with high income economies (according to per capita GNP) showed greater CGE. The size of the state had a strong influence on infant mortality $(r=0.40$; beta $=-1.327$; $E E=0.237 ; t=-5.590 ; p<0.001)$. Per capita GNP and region (especially Africa) were also associated with changes in health, coinciding with the worsening of health indicators in some poor countries of Africa and Asia. In the adjusted model, CGE maintained the statistically significant association with infant and maternal mortality.

Conclusion: The study suggests that the size of the state has a strong influence on health, especially on mortality. Although this study has certain limitations, given the reduced time window used, the results should be taken into consideration in the current political and epidemiologist debate.

Key words: Health. State. Public Expenditure. Globalization. Ecological study.
Correspondencia: Álvaro Franco. Departamento de Salud Pública. Universidad de Alicante. Apartado 99. Campus San Vicente del Raspeig. 03080 Alicante. España.

Correo electrónico: alvarofrancogiraldo @ hotmail.com.

\section{Introducción}

E I tamaño ideal del Estado siempre ha presentado dificultades de definición para los teóricos de la política social ${ }^{1,2}$. Recientes estudios lo miden como el porcentaje del gasto público con respecto al producto interior bruto $(\mathrm{PIB})^{3}$. Un sector de la opinión mundial ha insistido, desde hace más de 20 años, en la necesidad de reducirlo para hacer más eficiente su 
gestión y aumentar el rendimiento económico ${ }^{4}$. Diversos organismos económicos internacionales, como el Banco Mundial y el Fondo Monetario Internacional, han incorporado incluso requisitos de disminución del gasto público entre sus políticas 5 . Otros planteamientos sostienen que es más importante la calidad de los procesos que el propio tamaño del Estado ${ }^{6,7}$.

En la bibliografía se encuentran pocas referencias dedicadas al tamaño del Estado, downsizing and public sector. Al explorar las bases de datos Ecolit, Medline y Francis se observa que la información sobre disminución del sector público y salud en los últimos 10 años ([government expenditure OR public cost OR public expenditure] AND [public sector and health and downsizing]) se refiere a la crisis del Estado del Bienestar como consecuencia de la globalización y sus efectos sobre la reducción del sector público ${ }^{8}$, a los efectos de los cambios globales en la inestabilidad en el trabajo ${ }^{9}$, en las condiciones de inseguridad generadas y sus efectos en la salud ${ }^{10} \mathrm{o}$ en el aseguramiento respecto a la salud ${ }^{11}$; y de forma más específica, algunos trabajos retoman las implicaciones del recorte fiscal y del sector público en las reformas de las organizaciones de salud en algunos países ${ }^{12,13}$ y en los mecanismos implementados por las propias instituciones para hacer más eficiente el gasto ${ }^{14}$. Otros efectos estudiados sobre la reducción del Estado se refieren a consecuencias psicológicas ${ }^{15}$, a diferencias de género ${ }^{16}$, a consecuencias sobre la fuerza laboral y la economía y a las relaciones con los sectores público y privado ${ }^{17}$. Otros análisis, más generales y teóricos, apuntan la exploración de indicadores que puedan mostrar la influencia de la globalización, la liberalización financiera o la liberalización comercial respecto a la salud ${ }^{18}$.

Las ciencias sociales y la epidemiología deben apoyar la gestión de las políticas públicas mediante la exploración de las conexiones entre los indicadores de salud y los determinantes macroeconómicos, y la aportación de investigaciones para una toma de decisiones bien informada. Hay pocos estudios que den cuenta de los factores globales y sus vínculos con la salud. Así, tenemos sólo algunas aproximaciones importantes en el campo financiero, como la efectuada por la Comisión de Macroeconomía y Salud de la Organización Mundial de la Salud ${ }^{19}$.

La globalización corresponde a «los procesos en virtud de los cuales los Estados nacionales soberanos se entremezclan y rubrican mediante actores transnacionales y sus respectivas probabilidades de poder, orientaciones, identidades y entramados varios ${ }^{20}$, como anota Beck, para quien el mercado mundial desaloja o sustituye el quehacer político de las naciones. De ahí que la investigación en salud pública deba avanzar más en la búsqueda de indicadores que puedan relacionar, por un lado, los cambios del Estado o su componente público (consecuencia de la globalización) y, por otro, los cambios en la salud.

Tradicionalmente sólo se ha valorado el efecto del gasto público y privado en salud, así como del gasto en la educación. En la última década han sido frecuentes los estudios relacionados con el gasto público en la salud y las cuentas nacionales de salud ${ }^{21,22}$ o su relación con las reformas sectoriales sanitarias ${ }^{23}$, con metodologías contables para el desarrollo de la eficiencia de las organizaciones y del sector salud ${ }^{24,25}$, o con el aseguramiento en salud ${ }^{26}$. Sin embargo, algunos investigadores encuentran que el impacto del gasto público en salud es muy pequeño al compararlo con el de otros factores socioeconómicos, como ingreso per cápita, inequidad en la distribución del ingreso y nivel de educación ${ }^{27}$. Muy pocos, sin embargo, relacionan el gasto gubernamental total de un Estado o del gobierno central, o la reducción del sector público total, con sus efectos en la salud de la población, como se pretende aquí. De ahí que se plantee como objetivo determinar la relación entre el tamaño del Estado a través del gasto gubernamental central, medido como porcentaje del PIB, y los indicadores de salud en una muestra de países de todas las regiones del mundo en la última década del siglo $\mathrm{xx}$.

\section{Métodos}

Se realizó un estudio ecológico en el que se establecieron categorías de países que permitieran la comparación del gasto gubernamental central y del PIB per cápita (PIBpc) entre ellos, y que facilitara el análisis del comportamiento de los indicadores de salud en estos países en relación con los indicadores socioeconómicos y la región.

\section{Población y muestra}

Se consideró una muestra por conveniencia de 90 países (tabla 1), pertenecientes a todas las regiones del mundo, que formaban parte de un grupo con disponibilidad de información sobre GGC, en la década pasada $^{28}$. Se incluyeron 19 países desarrollados (D), 8 en transición económica (TE) y 63 en desarrollo; de éstos, 24 de Asia y Oceanía (AO), 21 de Latinoamérica y el Caribe (LAC) y 18 de África (A).

\section{Fuentes de información}

Los datos correspondientes al GGC y al PIBpc se obtuvieron del Informe de Expertos de Naciones Uni$\operatorname{das}^{28}$ y de las estadísticas oficiales del Fondo Moneta- 


\begin{tabular}{|c|c|c|c|c|}
\hline Desarrollados & En transición económica & Asia y Oceanía & América Latina y el Caribe & África \\
\hline Australia & Bielorrusia & Bhután & Argentina & Botswana \\
\hline Austria & Bulgaria & China & Bahamas & Burundi \\
\hline Canadá & República Checa & Chipre & Bolivia & República \\
\hline Dinamarca & Estonia & Fiji & Brasil & Democrática \\
\hline Finlandia & Hungría & India & Belice & Congo \\
\hline Francia & Lituania & Irán & Chile & Egipto \\
\hline Grecia & Polonia & Israel & Colombia & Etiopía \\
\hline Islandia & Rumania & Jordania & Costa Rica & Gambia \\
\hline Irlanda & & Kuwait & República Dominicana & Ghana \\
\hline Luxemburgo & & Líbano & Ecuador & Kenya \\
\hline Malta & & Malasia & El Salvador & Madagascar \\
\hline Holanda & & Myanmar & Granada & Mauritius \\
\hline Nueva Zelanda & & Nepal & Guatemala & Marruecos \\
\hline Noruega & & Omán & México & Namibia \\
\hline España & & Pakistán & Antillas Holandesas & Rwanda \\
\hline Suecia & & Filipinas & Nicaragua & Sierra Leona \\
\hline Suiza & & República Korea & Panamá & Sudáfrica \\
\hline Reino Unido & & Singapore & S. Vicente Grenada & Túnez \\
\hline \multirow[t]{6}{*}{ Estados Unidos } & & Sri Lanka & Trinidad y Tobago & Zambia \\
\hline & & R.A. Syria & Uruguay & Zimbabwe \\
\hline & & Tailandia & Venezuela & \\
\hline & & Turquía & & \\
\hline & & Emiratos Árabes & & \\
\hline & & Yemen & & \\
\hline
\end{tabular}

Fuente: Organización de las Naciones Unidas ${ }^{28}$.

rio Internacional ${ }^{29}$. Los indicadores de salud se obtuvieron del Programa de Naciones Unidas para el Desarrollo ${ }^{30}$ y de la Organización Mundial de la Salud. Se encontró información para estos indicadores entre 1990 y 2000, aunque la disponibilidad varía de un año a otro (los años con información más completa sobre GGC fueron 1990 y 1997). Primero se obtuvieron los datos de GGC y luego los de salud, para períodos respectivos.

\section{Indicadores}

El tamaño del Estado se mide a partir del GGC como porcentaje del PIB. El GGC incluye no sólo el gasto social (salud, educación, seguridad social, pensiones, subsidios), sino todo el gasto gubernamental, la inversión y las transferencias. También fueron considerados otros indicadores como el PIB pc (dólares americanos/habitante/año) y la región, por el efecto como potenciales variables de confusión.

La región se asimila en el estudio a la clasificación internacional de países según las condiciones socioeconómicas del mundo, descrita en el Informe de Expertos de Naciones Unidas ${ }^{28}$ : países desarrollados, en transición económica y en desarrollo, y países desagregados, estos últimos según la ubicación geográfica. Para salud se buscaron indicadores específicos: es- peranza de vida (en años), mortalidad infantil (por 1.000 nacidos vivos) y mortalidad materna (por 100.000 nacidos vivos).

\section{Análisis estadístico}

Con ayuda del paquete estadístico SPSS para Windows se realizó el análisis exploratorio del PIBpc y de la evolución del GGC como porcentaje del PIB. Esta última variable se midió de 2 formas en el estudio, como variable continua y como variable categórica. El tamaño del Estado, como variable categórica, se midió agrupando los países según el GGC («medianos», los que están alrededor de la media $(28 \%)$ del $G G C \pm 1$ desviación estándar (DE) ( $n=11)$; «pequeños», los que quedan por debajo de este rango, y «grandes» los que quedan por encima).

Los datos se analizaron al considerar globalmente todo el período y estimar relaciones del GGC con cada uno de los indicadores de salud de la población (variables dependientes), mediante coeficientes de correlación y de regresión lineal. Finalmente, se aplicó el modelo de regresión lineal múltiple para estimar el efecto del GGC sobre cada uno de los indicadores de salud de manera independiente (esperanza de vida, mortalidad infantil, mortalidad materna), efectuando valora- 
ciones de acuerdo con el coeficiente de determinación $\left(R^{2}\right)$. El PIBpc, el GGC y la región se introdujeron en el modelo siguiendo el método hacia atrás como variables continuas; la región se transformó en 4 variables artificiales, dada su condición cualitativa, y se consideró el paso de la región más desarrollada (D) a cada una de las demás. Por último, se analiza el comportamiento de los indicadores de salud comparados, por agrupaciones de países, según categorías de tamaño del Estado en cada una de las regiones.

En todos los casos en que se ajustaron los modelos de regresión lineal múltiple se evaluaron los supuestos y las condiciones de la regresión a partir del análisis de los residuos, la normalidad, la linealidad, la homocedasticidad y la falta de autocorrelación, requisitos que se cumplieron en la mayoría de los casos excepto para esperanza de vida, en la que fue necesario hacer transformaciones logarítmicas aunque los efectos variaron poco. También se aplicó el test de ANOVA de la regresión (véanse los estadísticos en el apartado Resultados).

\section{Resultados}

\section{Análisis descriptivo}

En la tabla 2 se presenta un resumen de los datos de los indicadores estudiados en los 90 países seleccionados. Los valores extremos de los indicadores de salud en el período estudiado fluctúan entre 3 (Suecia, Suiza) y 180 (Sierra Leona) para la mortalidad infantil, con una media de 39 por cada 1.000 nacidos vivos; entre 1 (Grecia) y 1.800 (Túnez) para la mortalidad materna, con una media de 216 por cada 1000.000 nacidos vivos; la esperanza de vida oscila entre 37 (Sierra Leona) y 79 (Suecia), con una media de 67 años.

Tabla 2. Comportamiento de los indicadores estudiados en 90 países, $1990-2000$

\begin{tabular}{lrrrrr}
\hline & GGC $^{\text {a }}$ & PIBpc $^{\text {b }}$ & $\begin{array}{c}\text { Mortalidad } \\
\text { infantic }\end{array}$ & $\begin{array}{c}\text { Mortalidad } \\
\text { maternad }^{d}\end{array}$ & $\begin{array}{c}\text { Esperanza } \\
\text { de vidae }^{\text {e }}\end{array}$ \\
\hline Media & 28 & 5717 & 39 & 216 & 67 \\
DE & 11 & 7573 & 37 & 348 & 11 \\
Mínimo & 6 & 97 & 3 & 1 & 37 \\
Máximo & 69 & 28114 & 180 & 1800 & 79 \\
\hline
\end{tabular}

GGC: gasto gubernamental central; PIBpc: producto interior bruto per cápita; DE: desviación estándar;

aGGC como porcentaje del PIB.

- PIBpc (en dólares de Estados Unidos/habitante/año.

'Tasa de mortalidad infantil: muertes en < 1 año por 1.000 nacidos vivos.

'Tasa de mortalidad materna: muertes maternas por cada 100.000 nacidos vivos. eEsperanza de vida: expectativa de vida al nacer, en años.
El GGC converge, al final del período, hacia una media del $28 \%$ ( $s=10,3$; cv $=36,8 \%$ ), pero aún dentro de un amplio rango $(7,8-53,5 \%)$ de países. Los valores extremos fluctúan en la década estudiada entre el 5,7\% de Sierra Leona y el 69,3\% de Kuwait. Entre los países con un GGC más alto (> 50\%) se encuentran Francia, Bulgaria, Holanda y Hungría, y entre los más bajos (<10\%) Nicaragua, China, Colombia, Myanmar, Guatemala y República del Congo.

Se encuentra una asociación del GGC con la región, con lo que se obtiene una dimensión diferente del Estado en los países ricos y menor en los países pobres. Si exploramos las economías de acuerdo con el PIBpc encontramos que los países ubicados en los niveles superiores de riqueza presentan mayor GGC.

Según los países, las diferencias en el PIBpc son grandes, con valores que fluctúan entre un mínimo de 97 dólares en República Democrática del Congo y un máximo de 28.114 dólares en Suiza, alrededor de una media de 5717 dólares en todo el período.

\section{Relación entre indicadores socioeconómicos y salud}

Se ha hallado una asociación entre las variables independientes GGC y PIBpc y los indicadores de salud. En una primera exploración realizada mediante correlaciones simples se supone una mayor relación del PIBpc que del GGC con los cambios en salud, aunque ambos muestran algún grado de correlación con éstos.

Al aplicar el modelo de regresión lineal simple a los datos agregados del período estudiado se han encontrado asociaciones estadísticamente significativas $(p<$ $0,001)$ entre el GGC y los indicadores de esperanza de vida $(r=0,30$; beta=0,283; $E E=0,069 ; t=4,120)$, la mortalidad infantil $(r=0,40$; beta $=-1,327$; $E E=0,237$; $\mathrm{t}=-5,590)$ y la mortalidad materna $(r=0,27$ : beta $=$ $-8,088$; $E E=2,419 ; t=3,343$ ).

Al ajustar los modelos de regresión lineal múltiple (tabla 3) observamos que para la mortalidad infantil, las 3 variables explicativas (GGC, PIB pc y región) presentan una asociación lineal significativa $(p<0,01)$. La región sólo resultó altamente significativa para el paso de los países desarrollados a los países de África. Para la mortalidad materna, al ajustar los efectos de las variables explicativas, se mantienen significativas en el modelo las asociaciones con la región (paso de los países desarrollados a África), el PIBpc y el GGC ( $p<0,01)$. Para la esperanza de vida, el coeficiente de determinación del modelo completo resultó ser el mayor del estudio, pero se pierde el efecto del GGC (coeficiente no significativo) y permanecen el efecto del PIBpc y el de la región (paso de los países desarrollados a África).

En la figura 1 se comparan los indicadores de salud, según las regiones y el tamaño del Estado, agregados para el período 1990-2000. De forma complementaria, 
Tabla 3. Tamaño del Estado y salud en 90 países (1990-2000).

\begin{tabular}{lcc}
\hline Modelos de regresión lineal ajustados por Producto Interior Bruto per capita y región & \\
\hline \multicolumn{1}{c}{ Indicador } & & Modelo $^{\mathrm{a}}$ \\
\cline { 2 - 3 } & Coeficiente de asociación $(\mathrm{B})$ & Coeficiente de determinación $\left(\mathrm{R}^{2}\right)$ \\
\hline Mortalidad infantil (por 1.000 nacidos vivos) & $-0.795 \cdot G G C^{*}$ & $\mathrm{R}^{2}=0.590(\mathrm{DW}=1.8 ; F=73.419 ; p<0,001)$ \\
Esperanza de vida (en años) & $+0.110 \cdot G G C$ & $R^{2}=0.632(D W=1.341 ; F=53.309 ; p<0,01)$ \\
Mortalidad materna (por 100.000 nacidos vivos) & $-5.695 \cdot G G C^{*}$ & $R^{2}=0.394(D W=1.847 ; F=16.899 ; p<0,001)$ \\
\hline
\end{tabular}

GGC: gasto gubernamental central

aLos coeficientes señalados con un asterisco* en el modelo tienen significación estadística $p<0,01$.

el estudio sugiere una mayor relación del GGC y los indicadores de salud en los países pobres.

\section{Discusión}

Los hallazgos fluctúan en la mayoría de países entre el 10 y el $40 \%$ del PIB, datos que coinciden con los del Banco Mundial ${ }^{5}$ y el Banco Interamericano de Desarr$\mathrm{Il}^{31}$. Lo más interesante es la tendencia de todas las regiones a converger hacia la media mundial, pero se mantiene la asociación del tamaño del Estado con la región, que es mayor en los países ricos y menor en los más pobres.

El tamaño del Estado examinado aquí, gracias a múltiples asociaciones del GGC, sugiere importantes conexiones del Estado y la salud, algunas de ellas en interacción con la región a la que pertenecen los países y con la distribución de la riqueza entre ellos, con evidentes disparidades que también se observan en otros análisis ${ }^{32}$.

La asociación de la región y del PIBpc con la salud coincide con el comportamiento histórico descrito por otros análisis ${ }^{33}$, pero en este caso se agrega la gran variabilidad entre países, relacionada posiblemente con el tamaño del Estado. En particular, el efecto del GGC (modelo de regresión lineal) tiene especial importancia en la mortalidad infantil, aunque también se encontró una elevada asociación con la mortalidad materna.

En el modelo de regresión lineal múltiple ajustado por el PIBpc y la región (tabla 3) se observa de forma más nítida el efecto del tamaño del Estado sobre salud: el aumento del GGC se asocia con un incremento de la esperanza de vida y con una disminución de la mortalidad infantil y materna; el cambio es mayor en este último indicador, además de ser estadísticamente significativo, al igual que en la mortalidad infantil. Aparte de otros análisis posibles, este solo hallazgo llevaría a recomendar acciones que condujeran a mejorar el gasto gubernamental para incidir de manera favorable en los indicadores sanitarios.
Sin embargo, los datos de la figura 1 muestran un efecto paradójico en los países con mayor tamaño del Estado (países desarrollados y países en transición económica): en ellos, la esperanza de vida muestra una relación no lineal con el tamaño del Estado que es menor en los Estados más grandes que en los de tamaño mediano. Esto nos puede sugerir, por un lado, que el aumento del tamaño del Estado no tiene un efecto uniforme en los indicadores de salud en todas las regiones y, por otro, que puede haber un tamaño óptimo del Estado a partir del cual las condiciones de salud pueden resultar negativamente afectadas. Se observa, además, una relación más favorable del tamaño del Estado y la salud en las regiones más pobres (mortalidad infantil en países de África y mortalidad materna en países en desarrollo).

No obstante, es necesario tener en cuenta que el tamaño del Estado incluye en su estructura factores como la educación, la salud y la seguridad social que se han asociado positivamente con la salud, pero también el gasto militar y otros factores que puede ser contraproducentes o pueden confundir el análisis.

Otros investigadores han denunciado los efectos funestos del recorte del Estado en los aspectos económicos, financieros y de salud, contraargumentando que es posible lograr crecimiento económico aunque se mantengan las políticas de equidad y paridad ${ }^{34,35}$. Otros autores concluyen que si los países reducen su gasto público ven lesionados sus indicadores básicos de salud y educación, y que estos cambios se asocian con las políticas de globalización ${ }^{36-38}$. En este sentido, resultará importante realizar otras investigaciones como la presente en las que se exploren también los efectos indirectos de la globalización, el tamaño del Estado y el Welfare state sobre la salud, vinculando otros sectores del bienestar y la macroeconomía, como ya lo han hecho algunos ${ }^{39}$.

En suma, hay razones para afirmar que el tamaño del Estado importa, en contraposición con el aforismo de que «el Estado mínimo es el Estado más extenso que puede ser justificado ${ }^{40}$. Para otros tratadistas, el Estado es el principal agente colectivo para garantizar el bienestar social, asegurar el acceso equitativo a los 
Figura 1. Indicadores de saluda según las regiones del mundo y el tamaño del Estadob, 1990-2000
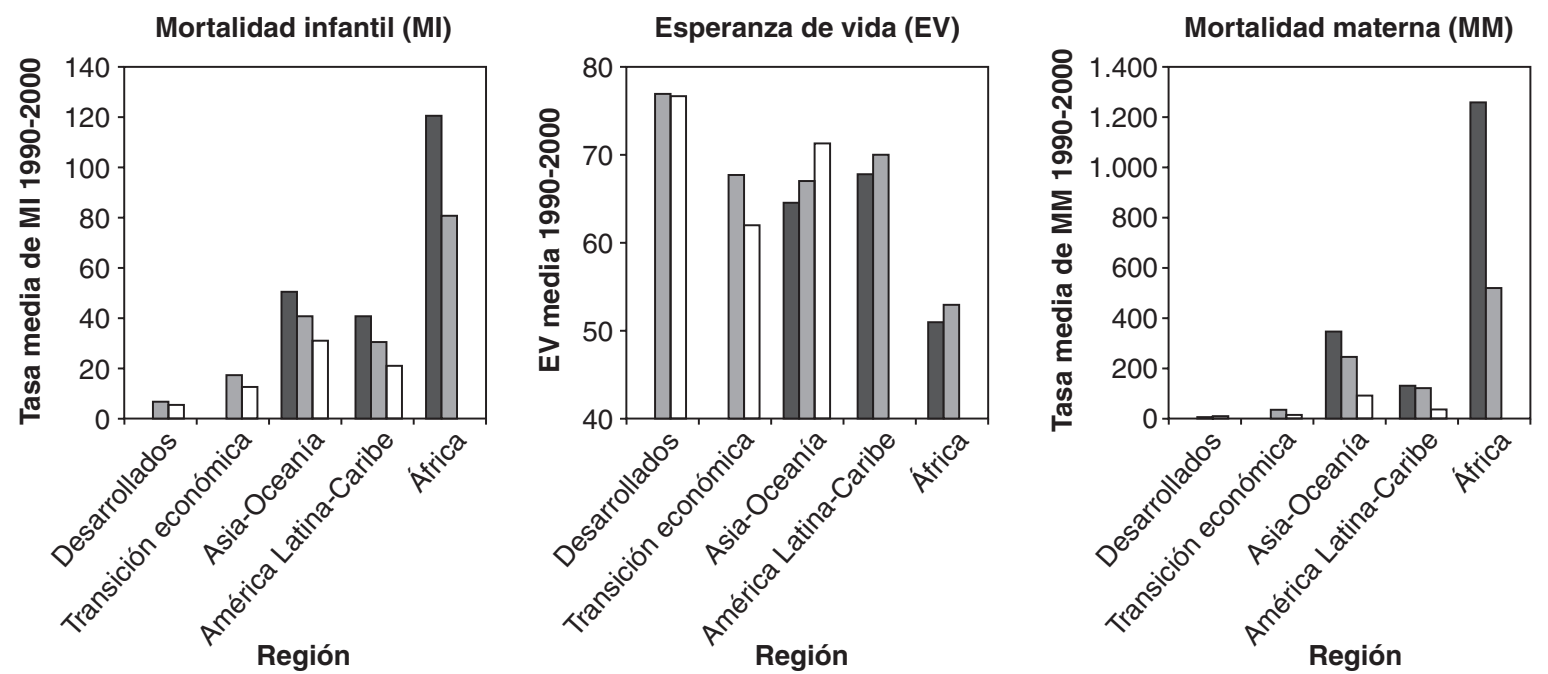

Tamaño estado
$\square$ Pequeños $\square$ Medianos $\square$ Grandes

aEsperanza de vida en años; mortalidada infantil por cada 1.000 nacidos vivos; mortalidad materna por cada 100.000 nacidos vivos.

bEquivalente al gasto gubernamental central, medido como porcentaje del producto interior bruto agrupado en 3 categorías.

servicios y combatir las barreras que lo obstaculizan ${ }^{41-}$ ${ }^{43}$, aunque su efectividad para resolver los problemas de la economía y la sociedad no sólo depende de su tamaño. La investigación de la salud puede hacer más evidente la relación de la dimensión del Estado con los indicadores sociales y de salud, no desvelada hasta el momento, y esclarecer más las posiciones políticas que se adopten en este campo.

En realidad, en este estudio hubo algunas limitaciones, como la reducida ventana temporal utilizada o no haber tenido en cuenta otras variables que pueden afectar al uso de los recursos públicos, como el pago del servicio de la deuda externa. Tampoco fue posible considerar, en esta etapa del estudio, la tipología de los Estados, que condiciona, sin duda, su tamaño y los efectos sobre la salud y el desarrollo. Para poder establecer una relación más precisa entre el gasto público y los indicadores sanitarios será necesario considerar, en un futuro, un período más amplio.

No hay datos suficientes de los indicadores básicos por países y para todos los años debido a un registro deficiente 0 a problemas de disponibilidad, aun tomándolos de las fuentes oficiales; esta situación es mucho más dramática para mortalidad materna ${ }^{44}$ y podría afectar en algún grado a los resultados.

No se puede olvidar que los estudios basados en medias nacionales esconden muchas e importantes diferencias regionales, subregionales e internas en los países en cuanto a sexos, etnia, capital social, clase social e ingresos que será necesario estudiar más a fondo. Igualmente, cabe esperar que una mayor desagregación de los datos internos por regiones o zona subnacionales permita obtener otro tipo de asociaciones del gasto gubernamental con la salud, que en este caso han podido quedar ocultas. La no inclusión en el estudio de algunos países del antiguo orbe socialista, como Cuba o Rusia, podría haber afectado de manera negativa a los resultados y haber disminuido el efecto esperado en la dirección de la hipótesis, ya que son estados de tamaño grande y, tradicionalmente, con buenos indicadores de salud.

En conclusión, las correlaciones estimadas reflejan un efecto importante del tamaño del Estado sobre la situación de salud, tanto si se analizan de manera independiente $o$ ajustadas por otras variables. A pesar de las relaciones cruzadas entre el gasto gubernamental, el PIBpc y la región, el modelo de regresión lineal múltiple muestra la asociación del tamaño del Estado con los indicadores de salud. Esta conclusión debe profundizarse mediante más investigaciones socials y aprovecharse para enriquecer el debate político y epidemiológico actual.

\section{Bibliografía}

1. Fleury S. Reforma del Estado. Revista Instituciones y Desarrollo. 2003;(14-15):81-122. 
2. Navarro V. Globalización económica, poder político y Estado del bienestar. 1. ${ }^{a}$ ed. Barcelona: Editorial Ariel S.A.; 2000.

3. United Nations. Group of experts Program in public administration and finance. Public Sector Indicators. Report prepared by the Secretariat [edición electrónica]. United Nations 2000 march 22. p. 1-17 [accedido 26 En 03]. Disponible en: http: //unpan1.un.org/intradoc/groups/public/documents/un.

4. Friedman T. Tradición versus innovación. 1. ${ }^{a}$ ed. Madrid: Atlántida; 1999.

5. Banco Mundial. Informe sobre el Desarrollo Mundial 1996. De la planificación centralizada a la economía de mercado. Washington DC: Banco Mundial; 1996.

6. Mann M. El futuro global del Estado-nación. Análisis Político. 1999;(38):3-18.

7. Centro Latinoamericano de Administración para el DesarroIlo (CLAD). Una Nueva Gestión Pública para América Latina. Documento preparado por el Consejo Científico del CLAD [edición electrónica]; 1998 [accedido 1 May 04]. Disponible en: http://unpan1.un.org/intradoc/groups/public/documents/CLAD/ UNPAN000161.pdf

8. Castles FG. The future of the welfare state: crisis myths and crisis realities. Int J Health Serv. 2002;32:255-77.

9. Wagar TH. Revue exploring the consequences of workforce reduction. Can J Administrative Sci. 1998;15:300-9.

10. Domenighetti G, D'Avanzo B, Bisig B. Health effects of job insecurity among employees in the Swiss general population. Int J Health Serv. 2000;30:477-90.

11. Fronstin P. Sources of health insurance and characteristics of the uninsured: analysis of the March 1999 Current Population Survey. EBRI Issue Brief. 2000;(217):1-26.

12. Acorn S, Crawford M. First line managers: scope of responsibility in a time of fiscal restraint. Healthc Manage Forum. 1996;9:26-30.

13. Bessler JS, Ellies M. Values and value a vision for the Australian health care system. Aust Health Rev. 1995;18:6-17.

14. Young S. Outsourcing and downsizing: processes of workplace change in public health. Econ Lab Relations Rev. 2002;13:244-69.

15. Campbell JF, Worrall LES, Cooper C, Greenglass ER, editors. Downsizing in Britain and its effects on survivors and their organizations; downsizing and restructuring: implications for stress and anxiety. Anxiety, Stress Coping. 2001;14:35-58.

16. Rama M. The gender implications of public sector downsizing: the reform program of vietnam. World Bank Res Observer. 2002;17:167-89.

17. Estache A, Laffont JJ, Zhang X. Downsizing with labor sharing and collusion. J Dev Econ. 2004;73:519-40.

18. Woodward D, Beaglehole R, Lipson D. La globalización de la salud: marco de análisis y de acción. Bull WHO 2002;6:327.

19. Feachem R. Commission on macroeconomics and health. WHO. Bull WHO. 2002;80:87.

20. Beck U. ¿Qué es la globalización? Falacias del globalismo, respuestas a la globalización. Barcelona: Ediciones Paidos Ibérica S.A.; 1998.

21. Musgrove P, Zeramdini R, Carrin G. Basic patterns in national health expenditure. Bull WHO. 2002;80:134-42.

22. Murray CJ, Govindaraj R, Musgrove P. National health expenditures: a global analysis. Bull WHO. 1994;72:623-37.

23. Figueras J, Musgrove P, Carrin G, Duran A. Retos para los sistemas sanitarios de Latinoamérica: que puede aprenderse de la experiencia europea? Gac Sanit. 2002;16:5-17.
24. Musgrove P. A critical review of «a critical review»: the methodology of the 1993 World Development Report, «Investing in Health». Health Policy Plan. 2000;15:110-5.

25. Musgrove P. La eficacia en función de los costos y la reforma del sector salud. Salud Publica Mex. 1995;37:363-74.

26. Musgrove P. Health insurance: the influence of the Beveridge Report. Bull WHO. 2000;78:845-6.

27. Filmer D, Pritchett $L$. The impact of public spending on health: does money matter? Soc Sci Med. 1999;(49):1309-23.

28. United Nations, Group of experts Program in public administration and finance. Annex. Central government expenditure as a percentage of GDP (table 2: domestic prices, 1990 and 1997). En: Report prepared by the Secretariat United Nations 2000 march 22 [edición electrónica] [accedido 26 En 03]. Disponible en: www.unpan.org/statiscal_database-publicsector.asp.

29. International Monetary Fund. International financial statistics yearbook 2000. Vol 53. Washington DC: IMF; 2000.

30. Programa de Naciones Unidas para el Desarrollo (PNUD). Informe de Desarrollo Humano; 1997.

31. Banco Interamericano de Desarrollo. América Latina frente a la desigualdad. Informe 1998-1999. Washington DC: BID; 1998. p. 222.

32. World Health Organization. The world health report- Health systems: Improving performance [edición electrónica]. Geneve: World Health Organization; 2001 [accedido 26 En 2003]. Disponible en: www.who.int/whr2001/2001/archives/2000/en/ press_release.htm.

33. Regidor E. Determinantes socioeconómicos de la salud. En: Regidor E, coordinador. Desigualdades sociales en salud: situación en España en los últimos años del siglo XX. Alicante: Universidad de Alicante; 2002. p. 13-35.

34. Siddiqi A, Hertzman C. Economic growth, income equality and population health among the Asian Tigers. Int J Health Serv. 2001;31:323-34.

35. Wilson K, Jerrett M, Eyles J. Testing relationships among determinants of health, health policy and self-assessed health status in Quebec. Int J Health Serv. 2001;31:67-90.

36. Weisbrot M, Baker D, Kraev E, Chen J. The scorecard on globalization 1980-2000: its consequences for economic and social well-being. Int J Health Serv. 2002;32:229-53.

37. Dollar D. ¿Es la globalización buena para la salud? Bull WHO. 2002;(6):16-9.

38. Andrea G. La globalización y la salud: resultados y opciones. Bull WHO. 2002;(6):23-31.

39. Navarro V, Shi L. The political context of social inequalities and health. Int J Health Serv. 2001;31:1-21.

40. Nozick R. Anarquía, Estado y utopía. Medellín: Fondo de Cultura Económica; 1988.

41. Habermas J. El valle de lágrimas de la globalización. Claves de Razón Práctica. 2001;109:5-10.

42. Klisberg B. ¿Cómo transformar el Estado? Más allá de mitos y dogmas. 2. ${ }^{a}$ ed. México: Fondo de Cultura Económica; 1992.

43. Almada C. Economic integration, development and public administration: the experiences of the European community and NAFTA. Toluca: IIAS; 1993.

44. Fondo de Población de las Naciones Unidas (UNFPA). Estado de la población mundial 2003 [versión electrónica]. Valorizar a 1000 millones de adolescentes. New York: UNFPA; 2003. p. 70-82. [accedido 15 Mar 2004]. Disponible en: 2003http://www.unfpa.org/swp/2003/presskit/pdf/indicators_spa.pdf. 